\title{
Precision of a Parabolic Optimum Calculated from Noisy Biological Data, and Implications for Quantitative Optimization of Biventricular Pacemakers (Cardiac Resynchronization Therapy)
}

\author{
Darrel P. Francis \\ International Centre for Circulatory Health, National Heart and Lung Institute, \\ Imperial College London, London, United Kingdom \\ E-mail:d.francis@imperial.ac.uk \\ Received August 26, 2011; revised November 9, 2011; accepted November 17, 2011
}

\begin{abstract}
In patients with heart failure and disordered intracardiac conduction of activation, doctors implant a biventricular pacemaker ("cardiac resynchronization therapy", CRT) to allow adjustment of the relative timings of activation of parts of the heart. The process of selecting the pacemaker timings that maximize cardiac function is called "optimization". Although optimization - more than any other clinical assessment-needs to be precise, it is not yet conventional to report the standard error of the optimum alongside its value in clinical practice, nor even in research, because no method is available to calculate precision from one optimization dataset. Moreover, as long as the determinants of precision remain unknown, they will remain unconsidered, preventing candidate haemodynamic variables from being screened for suitability for use in optimization. This manuscript derives algebraically a clinically-applicable method to calculate the precision of the optimum value of $x$ arising from fitting noisy biological measurements of $y$ (such as blood flow or pressure) obtained at a series of known values of $x$ (such as atrioventricular or interventricular delay) to a quadratic curve. A formula for uncertainty in the optimum value of $x$ is obtained, in terms of the amount of scatter (irreproducibility) of $y$, the intensity of its curvature with respect to $x$, the width of the range and number of values of $x$ tested, the number of replicate measurements made at each value of $x$, and the position of the optimum within the tested range. The ratio of scatter to curvature is found to be the overwhelming practical determinant of precision of the optimum. The new formulae have three uses. First, they are a basic science for anyone desiring time-efficient, reliable optimization protocols. Second, asking for the precision of every reported optimum may expose optimization methods whose precision is unacceptable. Third, evaluating precision quantitatively will help clinicians decide whether an apparent change in optimum between successive visits is real and not just noise.
\end{abstract}

Keywords: Cardiac, Pacemaker, Parabolic, Haemodynamics

\section{Introduction}

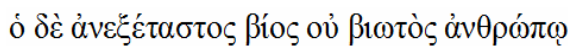
Plato, Apology of Socrates, 38a

Every year, $\sim 100,000$ cardiac resynchronization pacemakers are being implanted into patients with heart failure, because they deliver substantial symptomatic and survival benefits [1-3] by altering intracardiac timings. After implantation, the process of determining which timings to programme is described as "optimization" [4-6].
Responses of physiological variables to changes in pacemaker settings fit well to a parabola in the vicinity of the optimum [6,7]. Curve-fitting to calculate a clinical optimum has the advantage of permitting interpolation to settings which were not directly tested, and also avoids the problem that simply "picking the highest" leads to illusory optima, illusory increments in physiology from optimization, and illusory changes in optimum over time [8].

In clinical practice physiological measurements con- 
tain noise which can sometimes be substantial in comparison to the underlying signal, and which prevents the true underlying optimum being identified precisely even with curve fitting (Figure 1). The impact of such noise can be reduced by averaging multiple measurements, but this consumes resources such as time in a clinical environment or battery power if the measurements are conducted by the implanted device. It is therefore important to be able to calculate the precision of an optimum so that resource usage can be planned to be appropriate to achieve the clinically-required precision.

As well as needing to know how many replicates are required, doctors planning an optimization protocol also need guidance regarding what range of settings to cover during testing, and how coarsely or finely. Widening the range or making finer-grained measurements (i.e. at more closely-spaced intervals) increase the cost of the optimization process, and so are only justified if there is a clinically-valuable increase in precision of the optimum.

Finally, doctors caring for patients from day to day need to know the uncertainty of the optimum obtained this clinical optimization process. Without this knowledge, it is impossible to interpret apparent differences in optimum within an individual patient between one assessment method and another, or over time $[8,9]$ or after an operation or a heart attack.

This paper derives a simple formula for the uncertainty of the parabolically-defined optimal setting of a pacemaker, and presents practical implications for protocol design and for medical practice.

\section{Description of Method}

\subsection{Physiological Measurements with Noise}

The pacemaker setting may be adjusted over a wide range of values. Within individual patients, clinical information provides a priori a constrained range which contains all biologically plausible locations of the optimum for that patient. During the optimization procedure, the clinician acquires a series of pairs of values of the pacemaker setting and the corresponding physiological measurement. The first value, the pacemaker setting, has negligible uncertainty because it is programmed digitally. However the second value, the measured physiological variable, is not perfectly reproducible and therefore has an element of uncertainty.

Some choices of physiological variable, such as blood pressure, permit automatic acquisition, while others, such as Doppler velocity-time integral, typically require human involvement for each measurement. In practice the protocol is often to make more than one replicate measurement at each setting, and then to summarize the data
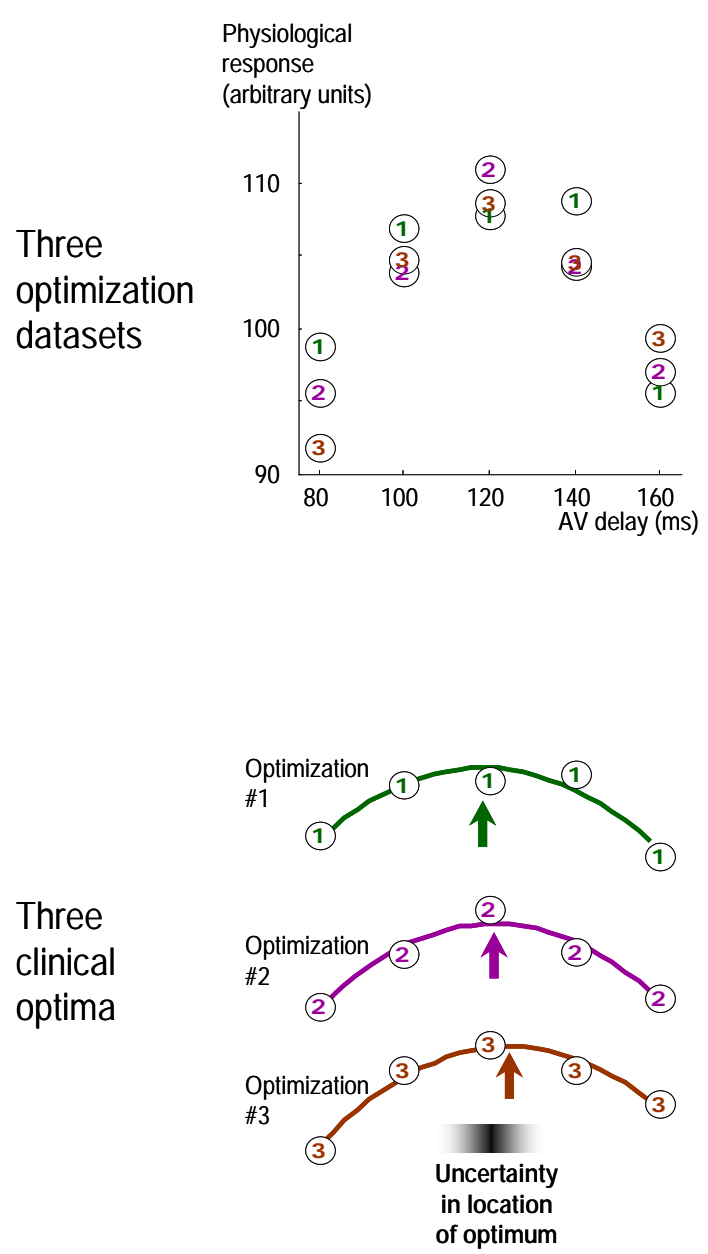

Figure 1. Sketch showing how noise in the measured data creates uncertainty in the optimum. Note: If there is enough time to conduct many optimizations, they can be analyzed separately to provide separate estimates of the optimum, so the uncertainty in the optimum can be observed. The upper panel sketches 3 optimization datasets in one simulated patient. All 3 datasets are plotted on the same graph and labeled " 1 " to " 3 ". The lower panel shows the 3 individual interpolated optima obtained by curve fitting with a parabola. This paper provides a method of calculating the uncertainty in a clinically-obtained optimum, without having to conduct several independent optimizations.

for each setting by the mean of the raw measurements at that setting.

Let the optimization protocol try $S$ different values $x_{i}$ of the pacemaker setting, evenly spaced at intervals of $\Delta x$. Let the averaged physiological measurements representing each setting be denoted $y_{i}$, each of which is the mean of $R$ replicate raw measurements. The observed data $y_{i}$ during clinical testing are composed of an underlying value $y_{u n d, i}$ and an error component $\varepsilon_{R}$.

$$
y_{i}=y_{\text {und }, i}+\varepsilon_{R}
$$

Let us consider the error component to be independent 
of $y_{u n d, i}$ and normally distributed, with its standard deviation for a single raw measurement being $\sigma_{\varepsilon_{1}}$ and, by the central limit theorem, the standard deviation for the error $\varepsilon_{R}$ of the average of $R$ replicates being $\sigma_{\varepsilon_{R}}=\sigma_{\varepsilon_{1}} / \sqrt{R}$.

\subsection{The Optimization Process}

The best-fit parabola, $A x_{i}^{2}+B x_{i}+C$, to the observed data is defined by the standard least-squares method, to minimize the squared deviation between the observations $y_{i}$ and the parabola. The clinical optimum from that fitted parabola is $-B / 2 A$, which will differ from the true optimum $O p t_{\text {True }}$ by an amount whose standard deviation can be calculated as follows. The best-fit parabola is defined by having $A, B$ and $C$ values that minimize the squared error $F=\sum\left(A x_{i}^{2}+B x_{i}+C-y_{i}\right)^{2}$.

This is achieved by setting all the partial derivatives of $F$ with respect to $A, B$ and $C$ to zero:

$$
\begin{gathered}
\partial F / \partial A=2 \sum\left[\left(A x_{i}^{2}+B x_{i}+C-y_{i}\right) x_{i}^{2}\right]=0 \\
\partial F / \partial B=2 \sum\left[\left(A x_{i}^{2}+B x_{i}+C-y_{i}\right) x_{i}\right]=0 \\
\partial F / \partial C=2 \sum\left[\left(A x_{i}^{2}+B x_{i}+C-y_{i}\right)\right]=0
\end{gathered}
$$

Therefore

$$
\begin{aligned}
A \sum x_{i}^{4}+B \sum x_{i}^{3}+C \sum x_{i}^{2} & =\sum x_{i}^{2} y_{i} \\
A \sum x_{i}^{3}+B \sum x_{i}^{2}+C \sum x_{i} & =\sum x_{i} y_{i} \\
A \sum x_{i}^{2}+B \sum x_{i}+C \sum 1 & =\sum y_{i}
\end{aligned}
$$

These can be solved for $A, B$ and $C$ as follows:

$$
\begin{aligned}
& A=\frac{S \sum x_{i}^{2} y_{i}-\sum x_{i}^{2} \sum y_{i}}{S \sum x_{i}^{4}-\left(\sum x_{i}^{2}\right)^{2}} \\
& B=\frac{\sum x_{i} y_{i}}{\sum x_{i}^{2}}, C=\frac{\sum x_{i}^{4} \sum y_{i}-\sum x_{i}^{2} \sum x_{i}^{2} y_{i}}{S \sum x_{i}^{4}-\left(\sum x_{i}^{2}\right)^{2}}
\end{aligned}
$$

\section{Results}

\subsection{The Clinical Optimum}

The clinician will choose as optimal the pacemaker setting which corresponds to the middle of the fitted parabola, i.e. $\hat{x}_{o p t}=\frac{-B}{2 A}$. In terms of the clinical data $x_{i}$ and $y_{i}$, this clinical optimum is

$$
\hat{x}_{\text {opt }}=\frac{\left(\sum x_{i}^{2}\right)^{2}-S \sum x_{i}^{4}}{2 S \sum x_{i}^{2} \sum x_{i}^{2} y_{i}-2 \sum x_{i}^{2} \sum y_{i}} .
$$

For simplicity, and without loss of generality, a coordinate system for $x$ can be chosen that makes 0 the centre of the range of settings tested during optimization. This symmetrical arrangement of $x_{i}$ values provides the following convenient identities:

$$
\begin{aligned}
& \sum x_{i}=0, \sum x_{i}^{2}=\Delta x^{2} S(S-1)(S+1) / 12, \\
& \sum x_{i}^{3}=0, \sum x_{i}^{4}=\Delta x^{4} S(S-1)\left(3 S^{2}-7\right)(S+1) / 240
\end{aligned}
$$

Applying these substitutions permits the clinical optimum to be described as follows:

$$
\hat{x}_{\text {opt }}=\frac{2\left(S^{2}-4\right) \Delta x^{2} \sum x_{i} y_{i}}{5\left(S^{2}-1\right) \Delta x^{2} \sum y_{i}-60 \sum x_{i}^{2} y_{i}} .
$$

If $y_{i}$ is augmented by any constant $k$, the numerator is unchanged because

$\sum x_{i}\left(y_{i}+k\right)=\sum x_{i} y_{i}+k \sum x_{i}=\sum x_{i} y_{i}$. The denominator is also unchanged because it is augmented by $5\left(S^{2}-1\right) \Delta x^{2} \sum k-60 k \sum x_{i}^{2} \quad$ which is $5 k S\left(S^{2}-1\right) \Delta x^{2}-\frac{60 k \Delta x^{2} S\left(S^{2}-1\right)}{12}=0$.

Therefore without loss of generality the observed values $y_{i}$ may be defined in terms of the underlying quadratic curvature coefficient $A_{u n d}$, the true optimum $x_{\text {opt }}$, and the noise component $\varepsilon_{R}$ as follows:

$$
\begin{gathered}
y_{i}=A_{\text {und }}\left(x_{i}-x_{\text {opt }}\right)^{2}+\varepsilon_{R, i} \\
\sum y_{i}=\sum A_{\text {und }}\left(x_{i}-x_{\text {opt }}\right)^{2}+\varepsilon_{R, i} \\
=A_{\text {und }}\left[\sum x_{i}^{2}-2 x_{\text {opt }} \sum x_{i}+S \cdot x_{\text {opt }}^{2}\right]+\sum \varepsilon_{R, i} \\
=A_{\text {und }} \Delta x^{2} \frac{S\left(S^{2}-1\right)}{12}+A_{\text {und }} \cdot S \cdot x_{\text {opt }}^{2}+\sum \varepsilon_{R, i} \\
\sum x_{i} y_{i}=\sum x_{i}\left[A_{\text {und }}\left(x_{i}-x_{\text {opt }}\right)^{2}+\varepsilon_{R, i}\right] \\
=A_{\text {und }}\left[\sum x_{i}^{3}-2 x_{\text {opt }} \sum x_{i}^{2}+x_{\text {opt }}^{2} \sum x_{i}\right]+\sum x_{i} \varepsilon_{R, i} \\
=-x_{\text {opt }} A_{\text {und }} \Delta x^{2} \frac{S\left(S^{2}-1\right)}{6}+\sum x_{i} \varepsilon_{R, i} \\
\sum x_{i}^{2} y_{i}=\sum x_{i}^{2}\left[A_{\text {und }}\left(x_{i}-x_{\text {opt }}\right)^{2}+\varepsilon_{R, i}\right] \\
=A_{\text {und }}\left[\sum x_{i}^{4}-2 x_{\text {opt }} \sum x_{i}^{3}+x_{\text {opt }}^{2} \sum x_{i}^{2}\right]+\sum x_{i}^{2} \varepsilon_{R, i} \\
=A_{\text {und }}\left[\Delta x^{4} \frac{S\left(S^{2}-1\right)\left(3 S^{2}-7\right)}{240}+x_{o p t}^{2} \Delta x^{2} \frac{S\left(S^{2}-1\right)}{12}\right] \\
+\sum x_{i}^{2} \varepsilon_{R, i}
\end{gathered}
$$

\subsection{Expression for the Clinical Optimum}

Applying these within Equation (1) gives: 


$$
\begin{aligned}
& \hat{x}_{\text {opt }}= \\
& 2\left(S^{2}-4\right) \Delta x^{2}\left[-x_{\text {opt }} A_{\text {und }} \Delta x^{2} \frac{S\left(S^{2}-1\right)}{6}+\sum x_{i} \varepsilon_{R, i}\right] \\
& 5\left(S^{2}-1\right) \Delta x^{2}\left[A_{\text {und }} \Delta x^{2} \frac{S\left(S^{2}-1\right)}{12}+A_{\text {und }} S x_{\text {opt }}^{2}+\sum \varepsilon_{R, i}\right]-60\left(A_{\text {und }}\left[\Delta x^{4} \frac{S\left(S^{2}-1\right)\left(3 S^{2}-7\right)}{240}+x_{\text {opt }}^{2} \Delta x^{2} \frac{S\left(S^{2}-1\right)}{12}\right]+\sum x_{i}^{2} \varepsilon_{R, i}\right) \\
& =\frac{2\left(S^{2}-4\right) \Delta x^{2}\left[-2 x_{\text {opt }} A_{\text {und }} \Delta x^{2} \frac{S\left(S^{2}-1\right)}{12}+\sum x_{i} \varepsilon_{R, i}\right]}{5\left(S^{2}-1\right) \Delta x^{2}\left[A_{\text {und }} \Delta x^{2} \frac{S\left(S^{2}-1\right)}{12}+\sum \varepsilon_{R, i}\right]-60 A_{\text {und }} \Delta x^{4} \frac{S\left(S^{2}-1\right)\left(3 S^{2}-7\right)}{240}-60 \sum x_{i}^{2} \varepsilon_{R, i}} \\
& =\frac{-A_{\text {und }} x_{\text {opt }} \Delta x^{4} \frac{S\left(S^{2}-1\right)\left(S^{2}-4\right)}{3}+2\left(S^{2}-4\right) \Delta x^{2} \sum x_{i} \varepsilon_{R, i}}{\Delta x^{4} \frac{5\left(S^{2}-1\right) S\left(S^{2}-1\right)}{12}-\Delta x^{4} \frac{S\left(S^{2}-1\right)\left(3 S^{2}-7\right)}{4}+\frac{5\left(S^{2}-1\right) \Delta x^{2}}{A_{\text {und }}} \sum \varepsilon_{R, i}-\frac{60}{A_{\text {und }}} \sum x_{i}^{2} \varepsilon_{R, i}} \\
& \hat{x}_{o p t}=\frac{-x_{o p t} \Delta x^{4} \frac{S\left(S^{2}-1\right)\left(S^{2}-4\right)}{3}+\frac{2\left(S^{2}-4\right) \Delta x^{2}}{A_{\text {und }}} \sum x_{i} \varepsilon_{R, i}}{-\Delta x^{4} \frac{S\left(S^{2}-1\right)\left(S^{2}-4\right)}{3}+\frac{5\left(S^{2}-1\right) \Delta x^{2}}{A_{\text {und }}} \sum \varepsilon_{R, i}-\frac{60}{A_{\text {und }}} \sum x_{i}^{2} \varepsilon_{R, i}} \\
& =\frac{x_{\text {opt }}-\frac{6}{A_{\text {und }} \Delta x^{2} S\left(S^{2}-1\right)} \sum x_{i} \varepsilon_{R, i}}{1-\frac{15}{A_{\text {und }} \Delta x^{2} S\left(S^{2}-4\right)} \sum \varepsilon_{R, i}-\frac{180}{A_{\text {und }} \Delta x^{4} S\left(S^{2}-1\right)\left(S^{2}-4\right)} \sum x_{i}^{2} \varepsilon_{R, i}}
\end{aligned}
$$

\subsection{Imprecision of the Clinical Optimum}

It can be seen from this that the clinically observed optimum $\hat{x}_{o p t}$ differs from the underlying optimum $x_{\text {opt }}$ because of two types of error, which affect the numerator and denominator respectively. The component in the numerator is a simple additive error. The impact of the denominator error, however, scales with $x_{o p t}$.

In clinical situations where the errors are large in comparison with the degree of curvature that is manifested, the entire denominator of this formula falls near (or beyond) zero and behavior of the expression becomes strongly nonlinear. In such a situation there is almost no useful information about the underlying optimum available from the acquired data. Clinically this can be considered likely whenever the information content (or intraclass correlation coefficient) is low [8].

In most situations of well-designed optimization protocols, however, the error is not large compared with the curvature of the signal, which makes it possible to provide a closed-form expression for the imprecision of the optimum.

To do this, the variance of the numerator is first shown to be

$$
\begin{aligned}
& \operatorname{Var}\left[\frac{6}{A_{\text {und }} \Delta x^{2} S\left(S^{2}-1\right)} \sum x_{i} \varepsilon_{R, i}\right] \\
& =\left(\frac{6}{A_{\text {und }} \Delta x^{2} S\left(S^{2}-1\right)} \sigma_{\varepsilon_{R}}\right)^{2} \sum_{i=-\frac{S-1}{2}}^{\frac{S-1}{2}} i^{2}=\frac{3}{A_{\text {und }}^{2} \Delta x^{2} S\left(S^{2}-1\right)} \sigma_{\varepsilon_{R}}^{2}
\end{aligned}
$$

Likewise the variance of the denominator is

$$
\begin{aligned}
& \operatorname{Var}\left[-\frac{15}{A_{\text {und }} \Delta x^{2} S\left(S^{2}-4\right)} \sum \varepsilon_{R, i}\right. \\
& \left.+\frac{180}{A_{\text {und }} \Delta x^{4} S\left(S^{2}-1\right)\left(S^{2}-4\right)} \sum x_{i}^{2} \varepsilon_{R, i}\right] \\
& =\operatorname{Var}\left[-\frac{15 \sum\left(S^{2}-1+12 i^{2}\right) \varepsilon_{R, i}}{A_{\text {und }} \Delta x^{2} S\left(S^{2}-1\right)\left(S^{2}-4\right)}+\right] \\
& =\frac{180}{A_{\text {und }}^{2} \Delta x^{4} S\left(S^{2}-1\right)\left(S^{2}-4\right)} \sigma_{\varepsilon_{R}}^{2}
\end{aligned}
$$


By the binomial expansion, $1 /(1-z)=1+z+z^{2}+z^{3}$ ‥ which can be approximated with $1+z$ as long as the magnitude of $z$ is well below 1 . Thus as long as we can make the following assumption:

$$
A_{\text {und }}^{2} \Delta x^{4} S\left(S^{2}-1\right)\left(S^{2}-4\right) \gg 180 \sigma_{\varepsilon_{R}}^{2}
$$

and as long as the numerator and denominator errors are not large, a linear approximation can be used for the variance of $\hat{x}_{o p t}$ as follows:

$$
\begin{aligned}
\operatorname{Var}\left(\hat{x}_{o p t}\right) \approx & \frac{3}{A_{\text {und }}^{2} \Delta x^{2} S\left(S^{2}-1\right)} \sigma_{\varepsilon_{R}}^{2} \\
& +x_{o p t}^{2} \cdot \frac{180}{A_{\text {und }}^{2} \Delta x^{4} S\left(S^{2}-1\right)\left(S^{2}-4\right)} \sigma_{\varepsilon_{R}}^{2} \\
& =\frac{\sigma_{\varepsilon_{R}}^{2}}{A_{\text {und }}^{2} \Delta x^{2}} \frac{3\left(S^{2}-4\right)+180 x_{o p t}^{2}}{S\left(S^{2}-1\right)\left(S^{2}-4\right)}
\end{aligned}
$$

The standard error of the clinical optimum, being the square root of this, is therefore

$$
S E\left(\hat{x}_{o p t}\right) \approx \frac{\sigma_{\varepsilon_{R}}}{A_{\text {und }} \Delta x} \frac{\sqrt{3} \sqrt{1+\frac{60}{S^{2}-4}\left(\frac{x_{o p t}}{\Delta x}\right)^{2}}}{\sqrt{S\left(S^{2}-1\right)}} .
$$

\subsection{Contributory Factors to Imprecision of the Clinical Optimum}

In general the optimization protocol may have multiple replicates, i.e. $R \geq 1$, in order to reduce the effect of noise. $\sigma_{\varepsilon_{R}}=\sigma_{\varepsilon_{1}} / \sqrt{R}$ so that in terms of the fundamental biological characteristics, the standard error of the clinical optimum is

$$
\begin{aligned}
& S E\left(\hat{x}_{\text {opt }}\right) \\
& \approx \frac{\sqrt{3}}{\sqrt{R \cdot S}} \cdot \frac{1}{\Delta x \sqrt{\left(S^{2}-1\right)}} \cdot \frac{\sigma_{\varepsilon_{1}}}{A_{\text {und }}} \cdot \sqrt{1+\frac{60}{S^{2}-4}\left(\frac{x_{o p t}}{\Delta x}\right)^{2}}
\end{aligned}
$$

This formulation highlights the 4 contributory factors to imprecision of the optimum clearly. First, the imprecision of the clinical optimum falls with the square root of $R \cdot S$, the number of individual physiological measurements made. Since making additional measurements either consumes scarce time in a clinical environment, or battery power if conducted automatically by the pacemaker, having knowledge of this tradeoff between number of measurements and imprecision may be helpful.

Second, by observing that the second term is almost the reciprocal of the width of the range of settings tested, $(S-1) \Delta x$, it can be seen that the wider the range tested, the more precise the optimum. In practice this is limited by loss of fit to the parabola for settings far from the optimum. However, within the range over which behaviour is parabolic, this analysis suggests it is desirable to cover a wide range rather than to focus exclusively on the very close vicinity of the optimum.

Third, biology sets an lower limit on $\frac{\sigma_{\varepsilon_{1}}}{A_{\text {und }}}$. While measurement error can be reduced by choosing measurement techniques with smaller instrument noise, eventually almost all the variability between replicates is genuine biological variation between heart beats, which places a lower boundary on $\sigma_{\varepsilon_{1}}$. Meanwhile $A_{\text {und }}$ is a manifestation of dependence of physiology upon changes in pacemaker setting, and is determined by the patient's own biological characteristics. For ideal measurement modalities, where the equipment contributes no noise, all the variability is biological, and may well be similar across different modalities. For example, measures of pressure and flow, although fundamentally different and having distinct units, may change proportionally with the same constant of proportionality in response to both signal (change in pacemaker setting) and noise (spontaneous biological variability). Thus $\frac{\sigma_{\varepsilon_{1}}}{A_{\text {und }}}$ may have a biologically-imposed lower limit within an individual patient which cannot be improved upon by the clinician.

The final term can be neglected if the optimum is very close to the centre of the tested range, i.e.

$\left|x_{o p t}\right| \ll \frac{S-1}{2} \Delta x$. However, it rises as $\left|x_{o p t}\right|$ rises. For

rapid interpretation by non-mathematicians it may be useful to develop a dimensionless variable $E$, representing how far the true optimum is away from the centre of the tested range, running from 0 when $x_{\text {opt }}=0$, to 1 when $\left|x_{\text {opt }}\right|=\frac{S-1}{2} \Delta x$.

Reworking Equation (3) to use this, and introducing $W$ $=(S-1) \Delta x$, the width of the range of settings tested, gives:

$$
S E\left(\hat{x}_{\text {opt }}\right) \approx \frac{\sqrt{3}}{\sqrt{R \cdot S}} \cdot \frac{1}{W} \frac{S-1}{\sqrt{S^{2}-1}} \cdot \frac{\sigma_{\varepsilon_{1}}}{A_{\text {und }}} \cdot \sqrt{1+15 \frac{(S-1)^{2}}{S^{2}-4} E^{2}}
$$

This formula is only valid when the condition described above in (2) is satisfied. That condition, the bare minimum number of raw measurements needed before the standard error of the optimum can be validly estimated, can be approximated in this simplified way:

$$
R \cdot S \gg 180\left(\frac{\sigma_{\varepsilon_{1}}}{W^{2} A_{\text {und }}}\right)^{2}
$$


If $R \cdot S$ exceeds the above formula by (for example) more than 5-fold, the uncertainty of the optimum is identified reliably using Equation (4). If $R \cdot S$ exceeds by only a small margin, Equation (4) will underestimate the observed scatter between repeat optimizations. Finally, if $R: S$ does not even exceed the right hand side, the dataset is so poor in information content that it should be discarded, and the protocol redesigned.

In practice the doctor does not know the underlying values of $\sigma_{\varepsilon_{1}}$ and $A_{\text {und }}$ but instead assesses them from clinical measurements: $\hat{\sigma}_{\varepsilon_{1}}$ and $\hat{A}$ respectively.

Figure 2 summarizes all the relevant variables in a format convenient for visual appreciation. It shows the steps necessary to gauge the uncertainty of the optimum.

The expressions can be applied to any unit of "pacemaker setting", and any unit of "physiological response". Typically pacemaker settings (atrioventricular and interventricular delay) are expressed in either milliseconds or seconds. Physiological response may be based on flow, for which suitable units might be true flow rates (e.g. $\mathrm{ml} / \mathrm{min}, \mathrm{L} / \mathrm{min}$ ) or expressed per beat (ml/beat), or as a peak instantaneous flow rate, or as an average velocity $(\mathrm{cm} / \mathrm{min}$ or $\mathrm{m} / \mathrm{min}$ ) or peak velocity, or velocity-time integral $(\mathrm{cm} /$ beat). Alternatively physiological response may be pressure (e.g. $\mathrm{mmHg}$ ) as a systolic, mean, or pulse pressure, or a value derived from pressure such as intraventricular peak first derivative of pressure $\left(\mathrm{d} p / \mathrm{d} t_{\max }\right)$. In principle uncalibrated physiological response variables and even those of unknown physical unit may be used, as long as it is reasonable to believe they vary approximately linearly with cardiac performance.

Any physical units may be used to express scatter $\sigma_{\varepsilon_{1}}$ and width $W$ but, once they are decided, the units that must be used to express curvature $A_{\text {und }}$ are [scatter]/ [width] $]^{2}$.

\subsection{Simplified Expression for Rapid Appreciation}

In practice the number of settings tested is usually fairly large, e.g. $\mathrm{S} \geq 6$, and $|E|$ does not exceed 1. Thus to explain the implications of the formula to a non-mathematician protocol designer, it may be sufficient to use the following simplified approximation. First check that $R \cdot S \gg 180\left(\frac{\hat{\sigma}_{\varepsilon_{1}}}{W^{2} \hat{A}}\right)^{2}$, otherwise redesign the experiment, with more replicates or with a variable which has a smaller $\hat{\sigma}_{\varepsilon_{1}} / \hat{A}$ ratio. Second:

$$
S E\left(\hat{x}_{\text {opt }}\right) \sim \frac{\sqrt{3}}{W \sqrt{R \cdot S}} \cdot \frac{\sigma_{\varepsilon_{1}}}{A_{\text {und }}} \sqrt{1+15 E^{2}}
$$

The left factor contains 3 variables known precisely from the experimental design. The right factor contains 3 variables that must be estimated from observations in the patient.

\subsection{Examples of Application to Existing Protocols}

\subsubsection{Example 1. Atrioventricular Delay Optimization}

In a published study [10] of clinical optimization in 15 patients, there were $S=6$ atrioventricular delay settings tested, and $R=6$ replicate measurements. The width of the spectrum of settings covered was $W=0.200-0.040$ $s=0.160 \mathrm{~s}$. The observed scatter between individual replicate measurements was $\hat{\sigma}_{\varepsilon_{1}}=3.9 \mathrm{mmHg}$. The observed curvature was $\hat{A}=1194 \mathrm{mmHg} \cdot \mathrm{s}^{-2}$. With these observed data, $180\left(\frac{\hat{\sigma}_{\varepsilon_{1}}}{W^{2} \hat{A}}\right)^{2}$ was 2.9 , which $R \cdot S$ comfortably exceeded.

For an optimum lying near the middle of the tested range, $E \approx 0$, so $S E\left(\hat{x}_{\text {opt }}\right) \approx \frac{\sqrt{3}}{\sqrt{6 \times 6}} \cdot \frac{1}{0.200} \cdot \frac{5}{\sqrt{35}} \cdot \frac{3.9}{1194} \approx 0.005$ s. For optima half-way to the edge of the tested spectrum, $S E\left(\hat{x}_{\text {opt }}\right)$ is almost double, at $0.010 \mathrm{~s}$. For optima at the edge of the tested spectrum, $S E\left(\hat{x}_{o p t}\right)$ is almost 4-fold higher than at the middle of the range, i.e. $\approx 0.019 \mathrm{~s}$.

Pacemakers only permit quantized values to be programmed, for example in steps of $0.010 \mathrm{~s}$. For optima lying near the centre of the tested range in that study, the optimization procedure can be seen to be sufficient to identify the optimum for clinical purposes. However, for optima at the edge of the tested range, the protocol would need to be adjusted to maintain that level of optimization precision. The most generically effective step to achieve this would be to conduct more replicate measurements.

\subsubsection{Example 2. Interventricular Delay Optimization}

In the same study, [10] optimization of interventricular delay was also examined. There were again $S=6$ settings tested, and $R=6$ replicate measurements. The width of the spectrum of settings covered was $W=0.120 \mathrm{~s}$. Observed measurement scatter $\hat{\sigma}_{\varepsilon_{1}}=3.9 \mathrm{mmHg}$. Curvature was measured to be $A_{\text {und }}=67 \mathrm{mmHg} \cdot \mathrm{s}^{-2}$. The bare minimum number of measurements needed

$180\left(\frac{\hat{\sigma}_{\varepsilon_{1}}}{W^{2} \hat{A}}\right)^{2}$ is $\sim 2900$, which $R \cdot S$ falls far below. This signals that application of Equation (4) will likely substantially underestimate the true standard error of the optimum.

Despite therefore being only a crude lower estimate, 


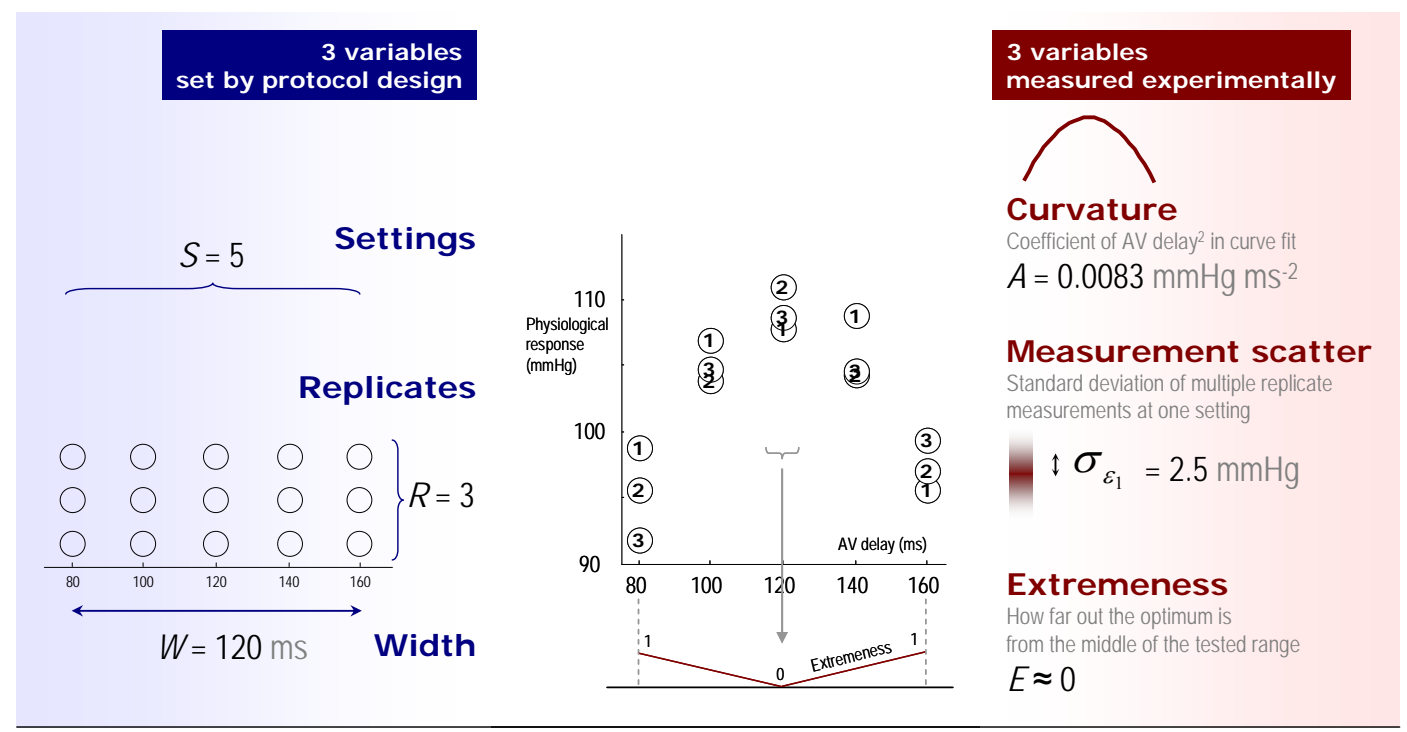

$\begin{aligned} & \text { Step } 1 . \text { To exclude grossly inadequate optimizations, check that } \\ & \text { In this case, } 15>>0.08 \text {, which is very satisfactory. }\end{aligned} \quad S>>180\left(\frac{\hat{\sigma}_{\varepsilon_{1}}}{W^{2} \hat{A}}\right)^{2}$

Step 2. Calculate standard error of optimum

$$
S E\left(\hat{x}_{o p t}\right) \approx \frac{\sqrt{3}}{\sqrt{R \cdot S}} \cdot \frac{1}{W} \frac{S-1}{\sqrt{S^{2}-1}} \cdot \frac{\hat{\sigma}_{\varepsilon_{1}}}{\hat{A}} \cdot \sqrt{1+15 \frac{(S-1)^{2}}{S^{2}-4} E^{2}} \approx 0.9 \mathrm{~ms}
$$

Figure 2. Illustration of the 6 variables that affect precision of the optimum. Note: Three are set by the design of the protocol and are therefore under the control of the clinician (within reason) and three are measured experimentally and depend on biology and on the physiological variable chosen for monitoring. Step 1 highlights the necessity for performing enough replicate measurements before attempting to calculate a standard error.

for an optimum lying in the middle of the tested range, Equation (4) provides $S E\left(\hat{x}_{\text {opt }}\right)>\sim 0.118 \mathrm{~s}$. For optima at the edge of the tested spectrum, $S E\left(\hat{x}_{\text {opt }}\right)$ is 4 -fold higher, i.e. $>\sim 0.422 \mathrm{~ms}$. These lower limits on the standard error are so large that the $95 \%$ confidence intervals $( \pm 1.96$ standard errors) dwarf the entire spectrum of clinically plausible settings.

This analysis shows that interventricular delay optimization conducted in this way in such patients is unreliable. Interventricular delay optimization protocols recommending a few (or even just one) measurement at each setting will never work. Biological variability has an ineradicable lower limit which can only be overcome by repetitions so numerous as to be unrealistic for manual methods such as echocardiography, and challenging even for automatable haemodynamic approaches.

The curious silence of the world literature on the elementary question of blinded test-retest reproducibility of interventricular delay optimization is a dog that didn't bark [11].

\subsection{Examples of Application in Protocol Design}

A doctor designing an optimization protocol can use this formula to ensure that the protocol delivers a clinicallysatisfactory degree of precision. Suppose the protocol needs to deliver optimization within $\pm 0.010 \mathrm{~s}$ on $95 \%$ of occasions, i.e. $S E\left(\hat{x}_{\text {opt }}\right)$ needs to be $0.005 \mathrm{~s}$. Rewriting Equation (4), we see that the number of replicate measurements required is:

$$
R \approx 3 \frac{(S-1)^{2}}{S^{3}-S}\left(\frac{1}{W \cdot S E\left(\hat{x}_{\text {opt }}\right)} \cdot \frac{\sigma_{\varepsilon_{1}}}{A_{\text {und }}}\right)^{2} \cdot\left(1+15 \frac{(S-1)^{2}}{S^{2}-4} E^{2}\right)
$$

A simplified form of the relationship, for easy appreciation by non-mathematicians, is shown below:

$$
R \sim \frac{3}{S}\left(\frac{1}{W \cdot S E\left(\hat{x}_{\text {opt }}\right)} \cdot \frac{\sigma_{\varepsilon_{1}}}{A_{\text {und }}}\right)^{2} \cdot\left(1+15 E^{2}\right)
$$

Three of the variables are handled easily. The protocol designer can choose how many settings to test $(S$, e.g. 6), and the width covered ( $W$, e.g. $0.160 \mathrm{~s})$ and can arrange from physiological knowledge that the optimum will lie in the middle $50 \%$ of the spectrum of tested settings, i.e. $E<1 / 2$.

The value of $\hat{\sigma}_{\varepsilon_{1}} / \hat{A}$ must be assessed experimentally 
for each proposed optimization variable. This requires only a few minutes per subject.

Table 1 shows how this ratio, and other variables, affect the number of replicates needed, $R$, to achieve the desired optimization precision.

Although mathematically the tested range $W$, the required precision $S E\left(\hat{x}_{o p t}\right)$, and the scatter-to-curvature ratio $\hat{\sigma}_{\varepsilon_{1}} / \hat{A}$ each have the same impact on the number of replicates required, in clinical practice $\hat{\sigma}_{\varepsilon_{1}} / \hat{A}$ has by far the greatest range of possible values, and the design process should therefore focus on ensuring that this is small, if the optimization protocol is to be practical.
The many haemodynamic variables that are affected by AV delay appear to be initially change proportionally [12] which suggests that $A_{\text {und }}$ may be approximately the same proportion of the baseline value of each variable. The protocol designer desiring small $\hat{\sigma}_{\varepsilon_{1}} / \hat{A}$ should therefore favour the variable whose scatter $\hat{\sigma}_{\varepsilon_{1}}$ is the smallest proportion of its baseline value.

\section{Limitations}

Although it is convenient to describe optimization responses with a parabola, the true underlying shape may

Table 1. Impact of physiology (scatter, curvature and extremeness) and protocol design (number of tested settings, width of spectrum) on the number of replicates needed for optimization of a desired level of precision.

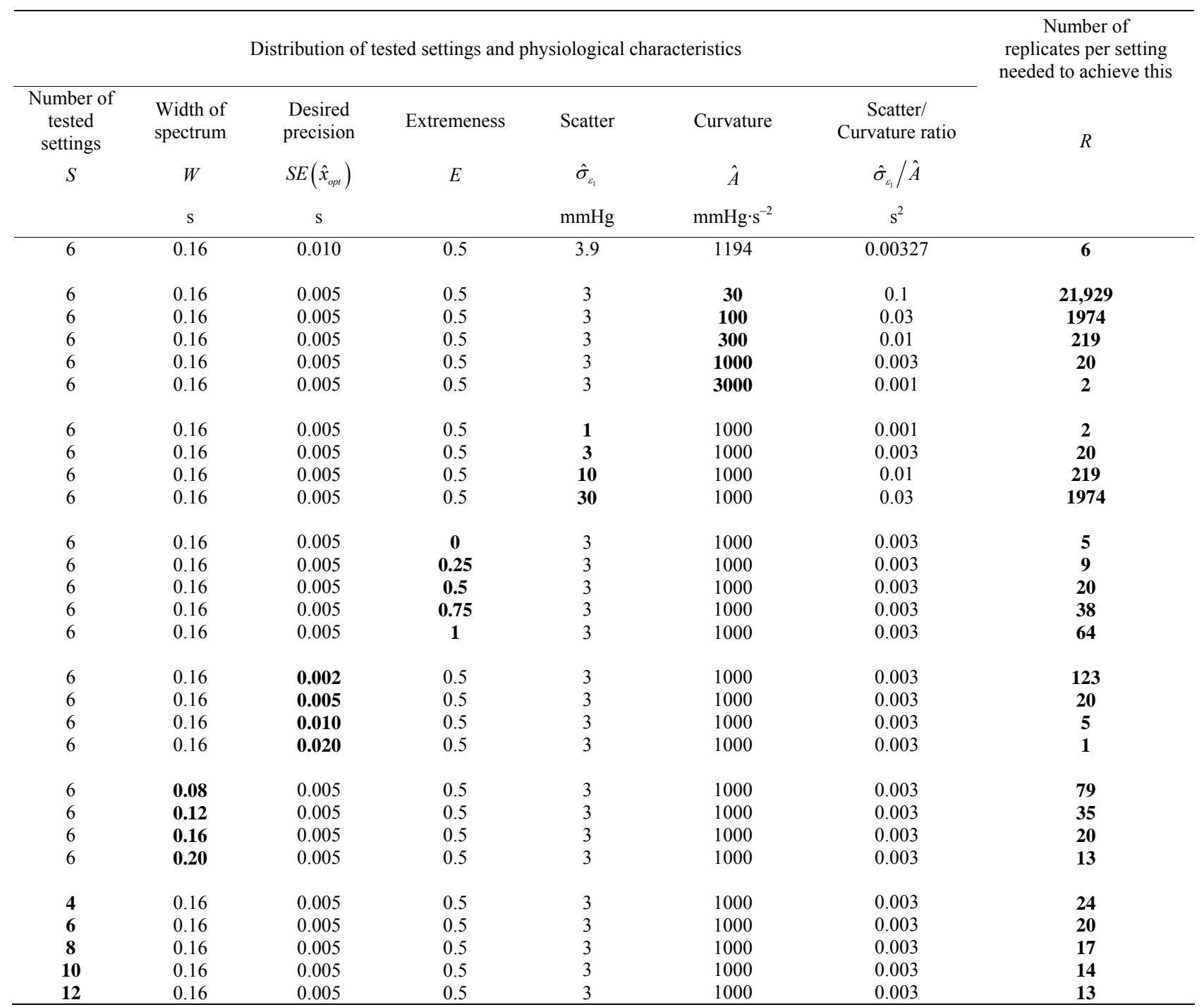

In practice the number of tested settings, width of spectrum, desired precision, and extremeness, all have a limited range of realistic values (as shown). In contrast, curvature and scatter have a wide range of possible values and, although rarely formally quantified in research reports, have enormous impact on the precision of the optimization process. This table can be used for physiological response measures that have any measurement unit (not only mmHg) since it is the relative size of scatter versus curvature, rather than their absolute values, that is important. For example, if "mmHg" was replaced by " $\%$ of value at reference setting", the table could without any other alteration be used for any variable such as echo-Doppler velocity-time integral, uncalibrated pressure signal, bioimpedance-derived stroke volume, or any marker of pulsatility in the peripheral circulation. 
be more complex. For example, where intrinsic (or even just fusion) conduction becomes active as AV delay is lengthened, the data points may deviate upwards from the parabolic trend, and so fitting to a parabola may bias the fitted AV delay optimum toward higher values. Nevertheless, the principles in this manuscript would still hold true. Moreover, in the close vicinity of the optimum, which is relevant to precise optimization, curvature may be closer to parabolic.

Merely applying formulae will not make optima more precise. Only selecting a physiological variable of suitably low $\hat{\sigma}_{\varepsilon_{1}} / \hat{A}$ ratio, and taking time to conduct enough measurements (shown in Equation (7)), can improve precision. Lack of interest in scatter and curvature in a doctor conducting optimization is as uninspiring as lack of interest in wings and engines in an aircraft pilot.

\section{Conclusions}

The practical implications may be summarized from inspection of each factor in Equation (6) in turn. To obtain precise clinical optima:

- most importantly, either commit enough resources to obtain enough measurements, or do not embark on optimization;

- cover a wide range of pacemaker settings while remaining within the parabolic region of the response curve;

- choose a physiological variable with as narrow a random variability (in relation to its sensitivity to pacemaker setting change) as possible; and

- if possible, design the spectrum of settings tested so that the true optimum will lie near its middle rather than at an extreme.

Clinicians treating patients and scientists designing and conducting studies have not had simple, quick methods to establish the uncertainty in planned or actual optimization procedures. As a result, patients may be undergoing apparent optimization procedures that are in fact worsening the programming of their pacemaker. Moreover without methods for recognizing unreliable optimizations, clinicians finding the optimum appearing to change every 1 or 2 years may feel compelled to carry out worthless optimization procedures more frequently, [8] which wastes clinical resources and may be harmful to patients.

The results in this paper permit easy quantification of uncertainty of the optimum of a cardiac resynchronization therapy pacemaker. They help patients gain the best physiological benefit, help doctors design protocols that deliver efficient and reliable optimization, and assist in distinguishing genuine change in patient physiology over time, versus random noise.
An unexamined optimization is not worth doing.

\section{Acknowledgements}

The author is supported by a Senior Research Fellowship (FS/10/038) from the British Heart Foundation.

\section{References}

[1] W. T. Abraham, W. G. Fisher and A. L. Smith, "Cardiac Resynchronization in Chronic Heart Failure," New England Journal of Medicine, Vol. 346, 2002, pp. 1845-1853. doi:10.1056/NEJMoa013168

[2] A. Kyriacou, P. A. Pabari, and D. P. Francis, "Cardiac Resynchronization Therapy Is Certainly Cardiac Therapy, But How Much Resynchronization and How Much Atrioventricular Delay Optimization?" Heart Failure Reviews, 2011, Article in Press. doi:10.1007/s10741-011-9271-1

[3] J. G. Cleland, J. C. Daubert, E. Erdmann, N. Freemantle, D. Gras, L. Kappenberger and L. Tavazzi, "Cardiac Resynchronization-Heart Failure (CARE-HF) Study Investigators. The Effect of Cardiac Resynchronization on Morbidity and Mortality in Heart Failure," New England Journal of Medicine, Vol. 352, No. 15, 2005, pp. 15391549. doi:10.1056/NEJMoa050496

[4] A. Auricchio, C. Stellbrink, S. Sack, M. Block, J. Vogt, P. Bakker, C. Huth, F. Schöndube, U. Wolfhard, D. Böcker, O. Krahnefeld and H. Kirkels, "Pacing Therapies In Congestive Heart Failure (PATH-CHF) Study Group. Long-Term Clinical Effect of Hemodynamically Optimized Cardiac Resynchronization Therapy in Patients With Heart Failure and Ven-Tricular Conduction Delay," Journal of the American College of Cardiology, Vol. 39, No. 12, 2002, pp. 2026-2033. doi:10.1016/S0735-1097(02)01895-8

[5] M. D. Bogaard, P. A. Doevendans, G. E. Leenders, P. Loh, R. N. Hauer, H. van Wessel and M. Meine, "Can Optimization of Pacing Settings Compensate for a NonOptimal Left Ventricular Pacing Site?" Europace, Vol. 12, No. 9, 2010, pp. 1262-1269. doi:10.1093/europace/euq167

[6] I. E. van Geldorp, T. Delhaas, B. Hermans, K. Vernooy, B. Broers, J. Klimusina, F. Regoli, F. F. Faletra, T. Moccetti, B. Gerritse, R. Cornelussen, J. Settels, H. J. Crijns, A. Auricchio and F. W. Prinzen, "Comparison of a NonInvasive Arterial Pulse Contour Technique and Echo Doppler Aorta Velocity-Time Integral on Stroke Volume Changes in Optimization of Cardiac Resynchronization Therapy," Europace, Vol. 13, No. 1, 2011, pp. 87-95. doi:10.1093/europace/euq348

[7] Z. I. Whinnett, J. E. Davies, G. Nott, K. Willson, C. H. Manisty, N. S. Peters, P. Kanagaratnam, D. W. Davies, A. D. Hughes, J. Mayet and D. P. Francis, "Efficiency, Reproducibility and Agreement of Five Different Hemodynamic Measures for Optimization of Cardiac Resynchronization Therapy," International Journal of Cardiology, Vol. 129, No. 2, 2008, pp. 216-226. 
doi:10.1016/j.ijcard.2007.08.004

[8] P. A. Pabari, K. Willson, B. Stegemann, I. E. van Geldorp, A. Kyriacou, M. Moraldo, J. Mayet, A. D. Hughes and D. P. Francis, "When Is an Optimization Not an Optimization? Evaluation of Clinical Impli-Cations of Information Content (Signal-to-Noise Ratio) in Optimization of Cardiac Resynchronization Therapy, and How to Measure and Maximize It," Heart Failure Reviews, Vol. 16, No. 3, 2011, pp. 277-290. doi:10.1007/s10741-010-9203-5

[9] W. T. Abraham, D. Gras, C. M. Yu, L. Guzzo and M. S. Gupta, "Rationale and Design of A Randomized Clinical Trial to Assess the Safety and Efficacy of Frequent Optimization of Cardiac Resynchronization Therapy: The Frequent Optimization Study Using the Quickopt Method (FREEDOM) Trial," American Heart Journal, Vol. 159, No. 6, 2010, pp. 944-948. doi:10.1016/j.ahj.2010.02.034

[10] Z. I. Whinnett, J. E. R. Davies, K. Willson, C. H. Manisty, A. C. Chow, R. A. Foale, D. W. Davies, A. D. Hughes, J.
Mayet and D. P. Francis, "Haemodynamic Effects of Changes in AV and VV Delay in Cardiac Resynchronization Therapy Show a Consistent Pattern: Analysis of Shape, Magnitude and Relative Importance of AV and VV Delay," Heart, Vol. 92, 2006, pp. 1628-1634. doi:10.1136/hrt.2005.080721

[11] A. C. Doyle, "The Curious Incident of the Dog in the Night-Time," Strand Magazine, Vol. 4, No. 4, 1892, pp. 645-660. http://www.archive.org/download/StrandMagazine24/ Strand24_text.pdf

[12] C. H. Manisty, A. Al-Hussaini, B. Unsworth, R. Baruah, P. A. Pabari, J. Mayet, A. D. Hughes, Z. I. Whinnett and D. P. Francis, "The Acute Effects of Changes to AV Delay on Blood Pressure and Stroke Volume: Potential Implications for Design of Pacemaker Optimization Protocols," Circulation: Arrhythmia and Electrophysiology, Article in Press. 